\title{
Comparison of non classical effects: quantum phase fluctuation, antibunching and minimum total noise in various non linear optical processes
}

\author{
Priyanka, Savita Gill* \\ Department of Applied Science, University Institute of Engineering and Technology, \\ Kurukshetra 136119, India \\ *savita2015@kuk.ac.in
}

DOI 10.17586/2220-8054-2020-11-2-161-170

\begin{abstract}
We study various non classical effects of light like reduction of quantum phase fluctuation, antibunching and minimum total noise present in various nonlinear optical processes. Further, we have shown that depth of non-classicality can be directly measured using all these parameters. We have done the comparative study to correlate these non-classical effects in seven wave mixing, eight wave mixing and third harmonic generation
\end{abstract}

Keywords: phase fluctuation, photon number, antibunching, minimum total noise.

Received: 26 January 2020

Revised: 20 February 2020

\section{Introduction}

For being a non-classical quantum state, negativities of Glauber-Sudarshan P-function may appear. However, this function may be strongly singular [1,2]. Generally we use standard deviation as the most natural measure to study quantum fluctuation [3]. Non classical effects of radiation field like reduction of quantum fluctuation, antibunching and minimum total noise were recently attracted a great interest in quantum optics. Quantum phase can be considered as a main feature to differentiate between classical and quantum physics. Antibunching and squeezing has no classical correlation and are called non classical states. In Sub-Poissonian (antibunching) photon statistics the variance of photon number is less than the mean value. The Hermitian quantum phase operators have some uncertainty [4-6] which leads to a lot of adverse formalism [7-9] of phase problem. Out of all these formalisms, Barnett-Pegg(BP) [8] and Susskind-Glogower(SG) [9] has contributed a considerable role in phase fluctuation and have been used by various researchers [10-18]. Study of phase fluctuation of coherent light coupled to a nonlinear medium and in intermediate state have already been studied $[19,20]$. The development of nanotechnology and nanoscience has provided new opportunities for nonlinear optics. In the last few decades, numerous studies on the nonlinear optical properties of novel materials have been performed because of the potential of these materials in optical device applications [21-25]. Strong optical nonlinearities in nanoparticles are observed due to quantum confinement, such as nonlinear optical absorption and second and third order optical nonlinearities; these can be studied for making optical modulators, optical limiters and laser second and third harmonic generators [23,24]. To study the optical nonlinearity of nanoparticles, there is a significant body of research concerning the measurements of third order nonlinear susceptibility $\chi^{3}$ which can be used as a source for the generation of third harmonic generation process [26]. With the role of quantum phase fluctuation in quantum cryptography [27], super conductivity [28,29] and with the success in experimental study of phase fluctuation [30], there has been a significant increase in importance of study of non-classical parameters.

In the present work, Carruther and Nieto phase parameters [16] are used to study phase fluctuation parameters in nonlinear processes like seven and eight wave interaction process and in third harmonic generation using BarnettPegg (BP) formalism [7]. We have reported in the paper that reduction of phase parameters not only directly implies antibunching but also increases with an increase in antibunching. We have also found minimum total noise in terms of number operators and observed that the greater the sub-Poissonian (antibunched) state, the greater will be the minimum total noise in the system.

\section{Measurement of quantum phase fluctuation parameters}

Barnett and Pegg [7] defined the exponential of phase operator $E$ and its Hermitian conjugate $E^{\dagger}$ as:

$$
\begin{aligned}
E & =\left(\bar{N}+\frac{1}{2}\right)^{-\frac{1}{2}} a(t), \\
E^{\dagger} & =\left(\bar{N}+\frac{1}{2}\right)^{-\frac{1}{2}} a^{\dagger}(t),
\end{aligned}
$$


where $\bar{N}$ is the mean photon number in the coherent state. Cosine and sine operators are defined as [7]:

$$
\begin{aligned}
& C=\frac{1}{2}\left(E+E^{\dagger}\right), \\
& S=-\frac{i}{2}\left(E-E^{\dagger}\right),
\end{aligned}
$$

and satisfy the following relations:

$$
\langle C\rangle^{2}+\langle S\rangle^{2}=1
$$

and

$$
[C, S]=\frac{i}{2}\left(\bar{N}+\frac{1}{2}\right)^{-\frac{1}{2}}
$$

and, we get

$$
(\Delta C)^{2}(\Delta S)^{2} \geq \frac{1}{16} \frac{1}{\left(\bar{N}+\frac{1}{2}\right)} .
$$

To measure quantum phase fluctuation, Carruthers and Nieto [16] had introduced $U, S$ and $Q$ parameters in the following way:

and

$$
\begin{gathered}
U\left(\theta, t,|\alpha|^{2}\right)=(\Delta N)^{2} \frac{(\Delta S)^{2}+(\Delta C)^{2}}{\langle S\rangle^{2}+\langle C\rangle^{2}} \\
S\left(\theta, t,|\alpha|^{2}\right)=(\Delta N)^{2}(\Delta S)^{2}
\end{gathered}
$$

$$
Q\left(\theta, t,|\alpha|^{2}\right)=\frac{S\left(\theta, t,|\alpha|^{2}\right)}{\langle C\rangle^{2}},
$$

where $\theta$ is the phase of input Poissonian state, $t$ is interaction time and $|\alpha|^{2}$ is mean photon number in coherent state.

\subsection{Seven wave interaction process}

In seven wave mixing process, the interaction takes place in such a way that the absorption of two photons of frequency $\omega_{1}$ each and the emission of two photons of frequency $\omega_{2}$ each and other two photons of frequency $\omega_{3}$. The Hamiltonian represents total energy for the system and is given as (taking $\hbar=1$ ):

$$
H=\omega_{1} a^{+} a+\omega_{2} b^{+} b+\omega_{3} c^{+} c+g\left(a^{2} b^{+2} c^{+3}+a^{+2} b^{2} c^{3}\right),
$$

where $g$ is a coupling constant, $a\left(a^{+}\right), b\left(b^{+}\right), c\left(c^{+}\right)$are the annihilation(creation) operators respectively. $A=a \exp i \omega_{1} t, B=b \exp i \omega_{2} t, C=c \exp i \omega_{3} t$ are slowly varying operators at frequencies $\omega_{1}, \omega_{2}$ and $\omega_{3}$.

To study quantum phase fluctuation, a coherent state $|\alpha\rangle$ is used as pump for mode $A$ and before the interaction process there was no photon in signal mode $B$ and stokes mode $C$ i.e.,

$$
|\psi\rangle=|\alpha\rangle|0\rangle|0\rangle .
$$

The Heisenberg equation of motion for fundamental mode $A$ is given as $(\hbar=1)$ :

$$
\frac{d A}{d t}=\frac{\partial A}{\partial t}+i[H, A]
$$

in this process, we assume the interaction time $t$ to be very small. Using short time approximation technique, expand $A(t)$ by using Taylor's series expansion and retain the terms up to second order in $g^{2} t^{2}$ :

$$
A(t)=A(0)+\frac{t}{1 !} \frac{d A(0)}{d t}+\frac{t^{2}}{2 !} \frac{d^{2} A(0)}{d t^{2}},
$$

and we get:

$$
\begin{gathered}
A(t)=A-2 i g t A^{\dagger} B^{2} C^{3}+g^{2} t^{2}\left(2 A N_{B}^{2} N_{C}^{3}-9 A^{\dagger} A^{2} N_{B}^{2} N_{C}^{2}-18 A^{\dagger} A^{2} N_{B}^{2} N_{C}\right. \\
-6 A^{\dagger} A^{2} N_{B}^{2}-4 A^{\dagger} A^{2} N_{B} N_{C}^{3}-36 A^{\dagger} A^{2} N_{B} N_{C}^{2}-72 A^{\dagger} A^{2} N_{B} N_{C}-24 A^{\dagger} A^{2} N_{B} \\
\left.-2 A^{\dagger} A^{2} N_{C}^{3}-18 A^{\dagger} A^{2} N_{C}^{2}-36 A^{\dagger} A^{2} N_{C}-12 A^{\dagger} A^{2}\right),
\end{gathered}
$$

where $N_{B}=B^{\dagger} B$ and $N_{C}=C^{\dagger} C$. 
From equation (12), the mean number of photons i.e. $N(t)=A^{\dagger}(t) \times A(t)$ can be expressed as:

$$
\begin{aligned}
N(t)=A^{\dagger} A-2 i g t( & \left.A^{\dagger 2} B^{2} C-A^{2} B^{\dagger 2} C^{\dagger}\right)+4 g^{2} t^{2}\left(2 A^{\dagger} A N_{B}^{2} N_{C}+N_{B}^{2} N_{C}\right) \\
- & 2 g^{2} t^{2}\left(A^{\dagger 2} A^{2} N_{B}^{2}+4 A^{\dagger 2} A^{2} N_{B} N_{C}+4 A^{\dagger 2} A^{2} N_{B}+2 A^{\dagger 2} A^{2} N_{C}+12 A^{\dagger 2} A^{2}\right),
\end{aligned}
$$

the expectation value of $N(t)$ using condition (10) is:

$$
\langle N\rangle=|\alpha|^{2}-24 g^{2} t^{2}|\alpha|^{4} .
$$

The squaring of expectation value of number operator is given as:

$$
\langle N\rangle^{2}=|\alpha|^{4}-48 g^{2} t^{2}|\alpha|^{6}+576 g^{4} t^{4}|\alpha|^{8},
$$

as the interaction time is small, we are taking terms up to $g^{2} t^{2}$ and above equation is written as:

$$
\begin{gathered}
\langle N\rangle^{2}=|\alpha|^{4}-48 g^{2} t^{2}|\alpha|^{6}, \\
\left\langle N^{2}\right\rangle=|\alpha|^{4}+|\alpha|^{2}-g^{2} t^{2}\left(48|\alpha|^{6}+96|\alpha|^{4}\right) .
\end{gathered}
$$

Using equation (15) and (16), we get:

$$
\begin{gathered}
(\Delta N)^{2}=\left\langle N^{2}\right\rangle-\langle N\rangle^{2}, \\
(\Delta N)^{2}=|\alpha|^{2}-g^{2} t^{2}\left(96|\alpha|^{4}\right) .
\end{gathered}
$$

The condition of sub-Poissonian photon statistics is given as:

$$
d=(\Delta N)^{2}-\langle N\rangle<0
$$

Using (14) and (17), we get:

$$
d=-72 g^{2} t^{2}|\alpha|^{4} .
$$

We obtained a negative value, which shows that the photon statistics is sub-Poissonian or antibunched light.

By substituting (13) in (2), we obtain:

$$
\begin{aligned}
C=\frac{1}{2}(N+ & \left.\frac{1}{2}\right)^{-\frac{1}{2}}\left[A+A^{\dagger}-2 i g t A^{\dagger} B^{2} C^{3}+2 i g t A B^{\dagger 2} C^{\dagger 3}+g^{2} t^{2}\left(2 A N_{B}^{2} N_{C}^{3}-9 A^{\dagger} A^{2} N_{B}^{2} N_{C}^{2}\right.\right. \\
- & 18 A^{\dagger} A^{2} N_{B}^{2} N_{C}-6 A^{\dagger} A^{2} N_{B}^{2}-4 A^{\dagger} A^{2} N_{B} N_{C}^{3}-36 A^{\dagger} A^{2} N_{B} N_{C}^{2}-72 A^{\dagger 2} A^{2} N_{B} N_{C} \\
& -24 A^{\dagger 2} A N_{B}-2 A^{\dagger} A^{2} N_{C}^{3}-18 A^{\dagger} A^{2} N_{C}^{2}-36 A^{\dagger} A^{2} N_{C}-12 A^{\dagger} A^{2}+2 A^{\dagger} N_{B}^{2} N_{C}^{3} \\
& -9 A^{\dagger 2} A N_{B}^{2} N_{C}^{2}-18 A^{\dagger 2} A N_{B}^{2} N_{C}-6 A^{\dagger 2} A N_{B}^{2}-4 A^{\dagger 2} A N_{B} N_{C}^{3}-36 A^{\dagger 2} A N_{B} N_{C}^{2} \\
& \left.\left.-72 A^{\dagger 2} A^{2} N_{B} N_{C}-24 A^{\dagger} A^{2} N_{B}-2 A^{\dagger 2} A N_{C}^{3}-18 A^{\dagger 2} A N_{C}^{2}-36 A^{\dagger 2} A N_{C}-12 A^{\dagger 2} A\right)\right]
\end{aligned}
$$

and

$$
\begin{aligned}
l S=-\frac{i}{2}(N+ & \left.\frac{1}{2}\right)^{-\frac{1}{2}}\left[A-A^{\dagger}-2 i g t A^{\dagger} B^{2} C^{3}-2 i g t A B^{\dagger 2} C^{\dagger 3}+g^{2} t^{2}\left(2 A N_{B}^{2} N_{C}^{3}-9 A^{\dagger} A^{2} N_{B}^{2} N_{C}^{2}\right.\right. \\
- & 18 A^{\dagger} A^{2} N_{B}^{2} N_{C}-6 A^{\dagger} A^{2} N_{B}^{2}-4 A^{\dagger} A^{2} N_{B} N_{C}^{3}-36 A^{\dagger} A^{2} N_{B} N_{C}^{2}-72 A^{\dagger 2} A^{2} N_{B} N_{C} \\
& -24 A^{\dagger 2} A N_{B}-2 A^{\dagger} A^{2} N_{C}^{3}-18 A^{\dagger} A^{2} N_{C}^{2}-36 A^{\dagger} A^{2} N_{C}-12 A^{\dagger} A^{2}-2 A^{\dagger} N_{B}^{2} N_{C}^{3} \\
+ & 9 A^{\dagger 2} A N_{B}^{2} N_{C}^{2}+18 A^{\dagger 2} A N_{B}^{2} N_{C}+6 A^{\dagger 2} A N_{B}^{2}+4 A^{\dagger 2} A N_{B} N_{C}^{3}+36 A^{\dagger 2} A N_{B} N_{C}^{2} \\
& \left.\left.+72 A^{\dagger 2} A^{2} N_{B} N_{C}+24 A^{\dagger} A^{2} N_{B}+2 A^{\dagger 2} A N_{C}^{3}+18 A^{\dagger 2} A N_{C}^{2}+36 A^{\dagger 2} A N_{C}+12 A^{\dagger 2} A\right)\right] .
\end{aligned}
$$

The expectation value of $C$ and $S$ operators of equation (20) and (21) by applying condition (10) are:

$$
\begin{aligned}
& \langle C\rangle=\frac{1}{2}\left[\left(N+\frac{1}{2}\right)^{-\frac{1}{2}}\left\{\alpha+\alpha^{*}-g^{2} t^{2}\left(12|\alpha|^{2} \alpha+12|\alpha|^{2} \alpha^{*}\right)\right\}\right], \\
& \langle S\rangle=-\frac{i}{2}\left[\left(\bar{N}+\frac{1}{2}\right)^{-\frac{1}{2}}\left\{\alpha-\alpha^{*}-g^{2} t^{2}\left(12|\alpha|^{2} \alpha-12|\alpha|^{2} \alpha^{*}\right)\right\}\right] .
\end{aligned}
$$

Then square of expectation value of $C$ and $S$ are:

$$
\langle C\rangle^{2}=\frac{1}{4}\left(N+\frac{1}{2}\right)^{-1}\left[\alpha^{2}+\alpha^{* 2}+2|\alpha|^{2}-24 g^{2} t^{2}\left(|\alpha|^{2} \alpha^{2}+|\alpha|^{2} \alpha^{* 2}+2|\alpha|^{4}\right)\right],
$$




$$
\langle S\rangle^{2}=-\frac{1}{4}\left(N+\frac{1}{2}\right)^{-1}\left[\alpha^{2}+\alpha^{* 2}-2|\alpha|^{2}-24 g^{2} t^{2}\left(|\alpha|^{2} \alpha^{2}+|\alpha|^{2} \alpha^{* 2}-2|\alpha|^{4}\right)\right] .
$$

Similarly:

$$
\begin{gathered}
\left\langle C^{2}\right\rangle=\frac{1}{4}\left(N+\frac{1}{2}\right)^{-1}\left[\alpha^{2}+\alpha^{* 2}+2|\alpha|^{2}+1-12 g^{2} t^{2}\left(\alpha^{2}+\alpha^{* 2}+2|\alpha|^{2} \alpha^{2}\right.\right. \\
\left.\left.+2|\alpha|^{2} \alpha^{* 2}+4|\alpha|^{4}+4|\alpha|^{2}\right)\right], \\
\left\langle S^{2}\right\rangle=-\frac{1}{4}\left(N+\frac{1}{2}\right)^{-1}\left[\alpha^{2}+\alpha^{* 2}-2|\alpha|^{2}-1-12 g^{2} t^{2}\left(\alpha^{2}+\alpha^{* 2}+2|\alpha|^{2} \alpha^{2}\right.\right. \\
\left.\left.+2|\alpha|^{2} \alpha^{* 2}-4|\alpha|^{4}-4|\alpha|^{2}\right)\right] .
\end{gathered}
$$

Using equations (24)-(27), second order variances is expressed as:

$$
(\Delta C)^{2}=\left\langle C^{2}\right\rangle-\langle C\rangle^{2}=\frac{1}{4}\left(N+\frac{1}{2}\right)^{-1}\left[1-12 g^{2} t^{2}\left(\alpha^{2}+\alpha^{* 2}+4|\alpha|^{2}\right)\right],
$$

and

$$
(\Delta S)^{2}=\left\langle S^{2}\right\rangle-\langle S\rangle^{2}=-\frac{1}{4}\left(\bar{N}+\frac{1}{2}\right)^{-1}\left[-1-12 g^{2} t^{2}\left(\alpha^{2}+\alpha^{* 2}-4|\alpha|^{2}\right)\right] .
$$

Now equations (6)-(8) can be expressed as:

and

$$
\begin{gathered}
U\left(\theta, t,|\alpha|^{2}\right)=\frac{1}{2}\left\{\frac{1-144 g^{2} t^{2}|\alpha|^{2}}{1-24 g^{2} t^{2}|\alpha|^{2}}\right\} \\
S\left(\theta, t,|\alpha|^{2}\right)=\frac{1}{4}\left(|\alpha|^{2}-24 g^{2} t^{2}|\alpha|^{4}+\frac{1}{2}\right)^{-1}\left[|\alpha|^{2}+24|\alpha|^{4} g^{2} t^{2}(\cos 2 \theta-6)\right]
\end{gathered}
$$

$$
Q\left(\theta, t,|\alpha|^{2}\right)=\frac{1+24|\alpha|^{2} g^{2} t^{2}(\cos 2 \theta-6)}{2(\cos 2 \theta+1)\left(1-24|\alpha|^{2} g^{2} t^{2}\right)} .
$$

From equation (19) and (30), it is clear that the reduction of phase parameter $U$ increases withan increase in the number of photons and is associated with antibunching.

\subsection{Eight wave interaction process}

In an eight wave mixing process, the interaction takes place in such a way that the absorption of two photons of frequency $\omega_{1}$ each and the emission of four photons of frequency $\omega_{2}$ each and another two photons of frequency $\omega_{3}$.

The Hamiltonian for the given process is:

$$
H=\omega_{1} a^{+} a+\omega_{2} b^{+} b+\omega_{3} c^{+} c+g\left(a^{2} b^{+4} c^{+2}+a^{+2} b^{4} c^{2}\right),
$$

where $g$ is a coupling constant, $a\left(a^{+}\right), b\left(b^{+}\right), c\left(c^{+}\right)$are the annihilation(creation) operators, respectively. $A=a \exp i \omega_{1} t, B=b \exp i \omega_{2} t, C=c \exp i \omega_{3} t$ are slowly varying operators at frequencies $\omega_{1}, \omega_{2}$ and $\omega_{3}$.

Now, using the short time approximation technique, Taylor's series expansion of $A(t)$ can be expressed as:

$$
\begin{gathered}
A(t)=A-2 i g t A^{\dagger} B^{4} C^{2}+g^{2} t^{2}\left(2 A N_{B}^{4} N_{C}^{2}-16 A^{\dagger} A^{2} N_{B}^{3} N_{C}^{2}-72 A^{\dagger} A^{2} N_{B}^{2} N_{C}^{2}\right. \\
-96 A^{\dagger} A^{2} N_{B} N_{C}^{2}-24 A^{\dagger} A^{2} N_{C}^{2}-4 A^{\dagger} A^{2} N_{B}^{4} N_{C}-64 A^{\dagger} A^{2} N_{B}^{3} N_{C}-288 A^{\dagger} A^{2} N_{B}^{2} N_{C} \\
-384 A^{\dagger} A^{2} N_{B} N_{C}-96 A^{\dagger} A^{2} N_{C}-2 A^{\dagger} A^{2} N_{B}^{4}-32 A^{\dagger} A^{2} N_{B}^{3}-144 A^{\dagger} A^{2} N_{B}^{2} \\
-192 A^{\dagger} A^{2} N_{B}-48 A^{\dagger} A^{2},
\end{gathered}
$$

where $N_{B}=B^{\dagger} B$ and $N_{C}=C^{\dagger} C$.

From equation (33), mean number of photons using condition (10) can be written as:

$$
N(t)=A^{\dagger} A-96 g^{2} t^{2}\left(A^{\dagger 2} A^{2}\right) .
$$

The expectation value of $N(t)$ is:

$$
\langle N\rangle=|\alpha|^{2}-96 g^{2} t^{2}|\alpha|^{4}
$$


Using equation (35), we get:

$$
(\Delta N)^{2}=|\alpha|^{2}-192 g^{2} t^{2}\left(2|\alpha|^{4}\right) .
$$

The condition of sub-Poissonian photon statistics is given as:

$$
d=(\Delta N)^{2}-\langle N\rangle<0
$$

Using equations (36) and (37), we get:

$$
d=-288 g^{2} t^{2}|\alpha|^{4}
$$

as $d$ is negative, and thus, we achieve an antibunched state.

Now, by substituting equation (34) in equation (2), we obtain:

$$
\begin{aligned}
C=\frac{1}{2}\left(N+\frac{1}{2}\right)^{-\frac{1}{2}}\left[A+A^{\dagger}-2 i g t A^{\dagger} B^{4} C^{2}+2 i g t A B^{\dagger 4} C^{\dagger 2}+g^{2} t^{2}\left(2 A N_{B}^{4} N_{C}^{2}\right.\right. \\
-16 A^{\dagger} A^{2} N_{B}^{3} N_{C}^{2}-72 A^{\dagger} A^{2} N_{B}^{2} N_{C}^{2}-96 A^{\dagger} A^{2} N_{B} N_{C}^{2}-24 A^{\dagger} A^{2} N_{C}^{2} \\
-4 A^{\dagger} A^{2} N_{B}^{4} N_{C}-64 A^{\dagger} A^{2} N_{B}^{3} N_{C}-288 A^{\dagger} A^{2} N_{B}^{2} N_{C}-384 A^{\dagger} A^{2} N_{B} N_{C} \\
-96 A^{\dagger} A^{2} N_{C}-2 A^{\dagger} A^{2} N_{B}^{4}-32 A^{\dagger} A^{2} N_{B}^{3}-144 A^{\dagger} A^{2} N_{B}^{2}-192 A^{\dagger} A^{2} N_{B} \\
-48 A^{\dagger} A^{2}+2 A^{\dagger} N_{B}^{4} N_{C}^{2}-16 A^{\dagger 2} A N_{B}^{3} N_{C}^{2}-72 A^{\dagger 2} A N_{B}^{2} N_{C}^{2}-96 A^{\dagger 2} A N_{B} N_{C}^{2} \\
-24 A^{\dagger 2} A N_{C}^{2}-4 A^{\dagger 2} A N_{B}^{4} N_{C}-64 A^{\dagger 2} A N_{B}^{3} N_{C}-288 A^{\dagger 2} A N_{B}^{2} N_{C}-384 A^{\dagger 2} A N_{B} N_{C} \\
\left.\left.-96 A^{\dagger 2} A N_{C}-2 A^{\dagger 2} A N_{B}^{4}-32 A^{\dagger 2} A N_{B}^{3}-144 A^{\dagger 2} A N_{B}^{2}-192 A^{\dagger 2} A N_{B}-48 A^{\dagger 2} A\right)\right]
\end{aligned}
$$

and

$$
\begin{gathered}
S=-\frac{i}{2}\left(N+\frac{1}{2}\right)^{-\frac{1}{2}}\left[A-A^{\dagger}-2 i g t A^{\dagger} B^{4} C^{2}-2 i g t A B^{\dagger 4} C^{\dagger 2}+g^{2} t^{2}\left(2 A N_{B}^{4} N_{C}^{2}-16 A^{\dagger} A^{2} N_{B}^{3} N_{C}^{2}\right.\right. \\
-72 A^{\dagger} A^{2} N_{B}^{2} N_{C}^{2}-96 A^{\dagger} A^{2} N_{B} N_{C}^{2}-24 A^{\dagger} A^{2} N_{C}^{2}-4 A^{\dagger} A^{2} N_{B}^{4} N_{C}-64 A^{\dagger} A^{2} N_{B}^{3} N_{C} \\
-288 A^{\dagger} A^{2} N_{B}^{2} N_{C}-384 A^{\dagger} A^{2} N_{B} N_{C}-96 A^{\dagger} A^{2} N_{C}-2 A^{\dagger} A^{2} N_{B}^{4}-32 A^{\dagger} A^{2} N_{B}^{3}-144 A^{\dagger} A^{2} N_{B}^{2} \\
-192 A^{\dagger} A^{2} N_{B}-48 A^{\dagger} A^{2}-2 A^{\dagger} N_{B}^{4} N_{C}^{2}+16 A^{\dagger 2} A N_{B}^{3} N_{C}^{2}+72 A^{\dagger 2} A N_{B}^{2} N_{C}^{2}+96 A^{\dagger 2} A N_{B} N_{C}^{2} \\
+24 A^{\dagger 2} A N_{C}^{2}+4 A^{\dagger 2} A N_{B}^{4} N_{C}+64 A^{\dagger 2} A N_{B}^{3} N_{C}+288 A^{\dagger 2} A N_{B}^{2} N_{C}+384 A^{\dagger 2} A N_{B} N_{C} \\
\left.\left.+96 A^{\dagger 2} A N_{C}+2 A^{\dagger 2} A N_{B}^{4}+32 A^{\dagger 2} A N_{B}^{3}+144 A^{\dagger 2} A N_{B}^{2}+192 A^{\dagger 2} A N_{B}+48 A^{\dagger 2} A\right)\right] .
\end{gathered}
$$

Using equations (40) and (41), second order variance is expressed as

$$
(\Delta C)^{2}=\left\langle C^{2}\right\rangle-\langle C\rangle^{2}=\frac{1}{4}\left(N+\frac{1}{2}\right)^{-1}\left[1-48 g^{2} t^{2}\left(\alpha^{2}+\alpha^{* 2}+4|\alpha|^{2}\right)\right]
$$

and

$$
(\Delta S)^{2}=\left\langle S^{2}\right\rangle-\langle S\rangle^{2}=-\frac{1}{4}\left(\bar{N}+\frac{1}{2}\right)^{-1}\left[-1-48 g^{2} t^{2}\left(\alpha^{2}+\alpha^{* 2}-4|\alpha|^{2}\right)\right] .
$$

Now, equations (6)-(8) can be expressed as:

$$
\begin{gathered}
U\left(\theta, t,|\alpha|^{2}\right)=\frac{1}{2}\left\{\frac{1-576 g^{2} t^{2}|\alpha|^{2}}{1-96 g^{2} t^{2}|\alpha|^{2}}\right\} \\
S\left(\theta, t,|\alpha|^{2}\right)=\frac{1}{4}\left(|\alpha|^{2}-96 g^{2} t^{2}|\alpha|^{4}+\frac{1}{2}\right)^{-1}\left[|\alpha|^{2}+96|\alpha|^{4} g^{2} t^{2}(\cos 2 \theta-6)\right],
\end{gathered}
$$

and

$$
Q\left(\theta, t,|\alpha|^{2}\right)=\frac{1+96|\alpha|^{2} g^{2} t^{2}(\cos 2 \theta-6)}{2(\cos 2 \theta+1)\left(1-96|\alpha|^{2} g^{2} t^{2}\right)} .
$$

From equation (38) and (44), we can correlate reduction of $\mathrm{U}$ with sub-Poissonian state. 


\subsection{Third harmonic generation process}

In third harmonic generation process, the interaction takes place in such a way that the absorption of three photons of frequency $\omega_{1}$ each and the emission of one photon of frequency $\omega_{2}$ such that $\omega_{2}=3 \omega_{1}$. The Hamiltonian for the given process is:

$$
H=\omega_{1} a^{+} a+\omega_{2} b^{+} b+g\left(a^{3} b^{+}+a^{+3} b\right),
$$

where $g$ is a coupling constant, $a\left(a^{+}\right), b\left(b^{+}\right)$are the annihilation(creation) operators, respectively. $A=a \exp i \omega_{1} t$, $B=b \exp i \omega_{2} t$ are slowly varying operators at frequencies $\omega_{1}, \omega_{2}$.

By using the same techniques used in above interaction process, we get;

$$
A(t)=A-3 i g t A^{\dagger 2} B+\frac{3}{2} g^{2} t^{2}\left(6 A^{\dagger} A^{2} N_{B}+6 A N_{B}-A^{\dagger 2} A^{3}\right),
$$

where $N_{B}=B^{\dagger} B$ and $N_{C}=C^{\dagger} C$.

From equation (48), the mean number of photons using condition (10) can be simplified as:

$$
N(t)=A^{\dagger} A-3 g^{2} t^{2}\left(A^{\dagger 3} A^{3}\right) .
$$

The expectation value of $N(t)$ is:

$$
\langle N\rangle=|\alpha|^{2}-3 g^{2} t^{2}|\alpha|^{6}
$$

Using equation (48), we get:

$$
(\Delta N)^{2}=|\alpha|^{2}-18 g^{2} t^{2}\left(|\alpha|^{6}\right) .
$$

The condition of sub-Poissonian photon statistics is given as:

$$
d=(\Delta N)^{2}-\langle N\rangle<0 \text {. }
$$

Using (50) and (51), we get:

$$
d=-15 g^{2} t^{2}|\alpha|^{6},
$$

as $d$ is negative and thus we achieve a non-classical state.

By substituting (48) in (2), we obtain:

$$
C=\frac{1}{2}\left(N+\frac{1}{2}\right)^{-\frac{1}{2}}\left[A+A^{\dagger}-3 i g t A^{\dagger 2} B+3 i g t A^{3} B^{\dagger}-\frac{3}{2} g^{2} t^{2}\left(A^{\dagger 2} A^{3}+A^{\dagger 3} A^{2}\right)\right]
$$

and

$$
S=-\frac{i}{2}\left(\bar{N}+\frac{1}{2}\right)^{-\frac{1}{2}}\left[A-A^{\dagger}-3 i g t A^{\dagger 2} B-3 i g t A^{2} B^{\dagger}-\frac{3}{2} g^{2} t^{2}\left(A^{\dagger 2} A^{3}-A^{\dagger 3} A^{2}\right)\right],
$$

Using equations (54)-(55), second order variance is expressed as:

$$
(\Delta C)^{2}=\left\langle C^{2}\right\rangle-\langle C\rangle^{2}=\frac{1}{4}\left(N+\frac{1}{2}\right)^{-1}\left[1-\frac{3}{2} g^{2} t^{2}\left(2|\alpha|^{2} \alpha^{2}+2|\alpha|^{2} \alpha^{* 2}+6|\alpha|^{4}\right)\right],
$$

and

$$
(\Delta S)^{2}=\left\langle S^{2}\right\rangle-\langle S\rangle^{2}=-\frac{1}{4}\left(\bar{N}+\frac{1}{2}\right)^{-1}\left[-1-\frac{3}{2} g^{2} t^{2}\left(2|\alpha|^{2} \alpha^{2}+2|\alpha|^{2} \alpha^{* 2}-6|\alpha|^{4}\right)\right] .
$$

Now equations (6)-(8) can be expressed as:

$$
\begin{gathered}
U\left(\theta, t,|\alpha|^{2}\right)=\frac{1}{2}\left\{\frac{1-27 g^{2} t^{2}|\alpha|^{4}}{1-3 g^{2} t^{2}|\alpha|^{4}}\right\}, \\
S\left(\theta, t,|\alpha|^{2}\right)=\frac{1}{4}\left(|\alpha|^{2}-3 g^{2} t^{2}|\alpha|^{6}+\frac{1}{2}\right)^{-1}\left[|\alpha|^{2}+3|\alpha|^{6} g^{2} t^{2}(2 \cos 2 \theta-9)\right],
\end{gathered}
$$

and

$$
Q\left(\theta, t,|\alpha|^{2}\right)=\frac{1+3|\alpha|^{4} g^{2} t^{2}(2 \cos 2 \theta-9)}{2(\cos 2 \theta+1)\left(1-3|\alpha|^{4} g^{2} t^{2}\right)},
$$

equations (53) and (58) indicate the presence of non-classicality. 


\section{Minimum total noise in terms of number operator}

The minimum total noise of a single mode with density matrix $\rho$ can be stated in terms of $A$ and $A^{\dagger}$ as [31]:

$$
X_{1}=\frac{1}{2}\left[A+A^{\dagger}\right] \quad \text { and } \quad X_{2}=\frac{1}{2 i}\left[A-A^{\dagger}\right] .
$$

From equation (60), we get:

$$
\left\langle X_{1}^{2}\right\rangle+\left\langle X_{2}\right\rangle^{2}=\left\langle N+\frac{1}{2}\right\rangle
$$

In order to find out the minimum total noise of state in terms of number operator we use Schwartz inequality:

$$
\left|\left\langle\left(X_{2}-\left\langle X_{2}\right\rangle\right)(N-\langle N\rangle)\right\rangle\right|^{2} \leq\left\langle\left(X_{2}-\left\langle X_{2}\right\rangle\right)^{2}\right\rangle\left\langle(N-\langle N\rangle)^{2}\right\rangle \leq\left(\Delta X_{2}\right)^{2}(\Delta N)^{2} .
$$

Equation (63) is simplified as:

$$
\left(\Delta X_{2}\right)^{2}(\Delta N)^{2} \geq \frac{1}{4}\left|\left\langle\left[X_{2}, N\right]\right\rangle\right|^{2} \geq \frac{1}{4}\left|\left\langle X_{1}\right\rangle\right|^{2}
$$

where $\left[X_{2}, N\right]=i X_{1}$.

Similarly,we can achieve for $X_{1}$ is:

$$
\left(\Delta X_{1}\right)^{2}(\Delta N)^{2} \geq \frac{1}{4}\left|\left\langle X_{2}\right\rangle\right|^{2}
$$

Using equation (64) and (65), we get:

$$
\left\langle X_{1}\right\rangle^{2}+\left\langle X_{2}\right\rangle^{2} \leq 4(\Delta N)^{2}\left[\left(\Delta X_{1}\right)^{2}+\left(\Delta X_{2}\right)^{2}\right] .
$$

Using equation (62), we obtain:

$$
\left\langle N+\frac{1}{2}\right\rangle \leq\left[4(\Delta N)^{2}+1\right]\left[\left(\Delta X_{1}\right)^{2}+\left(\Delta X_{2}\right)^{2}\right],
$$

thus minimum total noise in terms of number operator is:

$$
T_{\min }=\left[\left(\Delta X_{1}\right)^{2}+\left(\Delta X_{2}\right)^{2}\right]
$$

Substituting equation (67) in above relation, we get:

$$
T_{\min } \geq \frac{\left\langle N+\frac{1}{2}\right\rangle}{\left[4(\Delta N)^{2}+1\right]},
$$

equation (68) shows that as the value of $(\Delta N)^{2}$ decreases, minimum total noise increases.

\subsection{Seven wave mixing process}

Minimum total noise in seven wave interaction process can be obtained by substituting equation (14) and (17) in equation (68):

$$
T_{N} \geq \frac{|\alpha|^{2}-24 g^{2} t^{2}|\alpha|^{4}+\frac{1}{2}}{4|\alpha|^{2}+1-384 g^{2} t^{2}|\alpha|^{4}} .
$$

\subsection{Eight wave mixing process}

In this case we substitute equation (36) and (37) in equation (68) to obtain the expression for minimum total noise and is given as:

$$
T_{N} \geq \frac{|\alpha|^{2}-96 g^{2} t^{2}|\alpha|^{4}+\frac{1}{2}}{4|\alpha|^{2}+1-1536 g^{2} t^{2}|\alpha|^{4}} .
$$

\subsection{Third harmonic generation process}

After substituting equation (50) and (51) in equation (68), we get expression of minimum total noise as:

$$
T_{N} \geq \frac{|\alpha|^{2}-3 g^{2} t^{2}|\alpha|^{6}+\frac{1}{2}}{4|\alpha|^{2}+1-72 g^{2} t^{2}|\alpha|^{6}},
$$

thus equations (69)-(71) show that for a fixed value of $\langle N\rangle$ as the value of $[\Delta N]^{2}$ decreases, minimum total noise increases which indicates the presence of quantum state. 


\section{Conclusion}

Comparison of non-classical parameters like phase fluctuation, antibunching and minimum total noise have been done in seven and eight wave mixing interaction and third harmonic generation nonlinear optical processes. Figs. 14 show that reduction of phase fluctuation parameters $U, S, Q$ and antibunching increases with increasing mean photon number $|\alpha|^{2}$. After comparing Figs. 1-4, we can conclude that rate of reduction of parameters $U, S, Q$ and antibunching is maximum in third harmonic generation as compared to eight wave mixing and seven wave mixing. Equations (30)-(32), (44)-(46), (58)-(60) show that the parameter $U$ is independent of $\theta$ while $S$ and $Q$ parameters can be tuned by varying the values of $t$ and $\theta$. The result also indicates that higher the rate of reduction of phase fluctuation parameter, higher will be the antibunching.

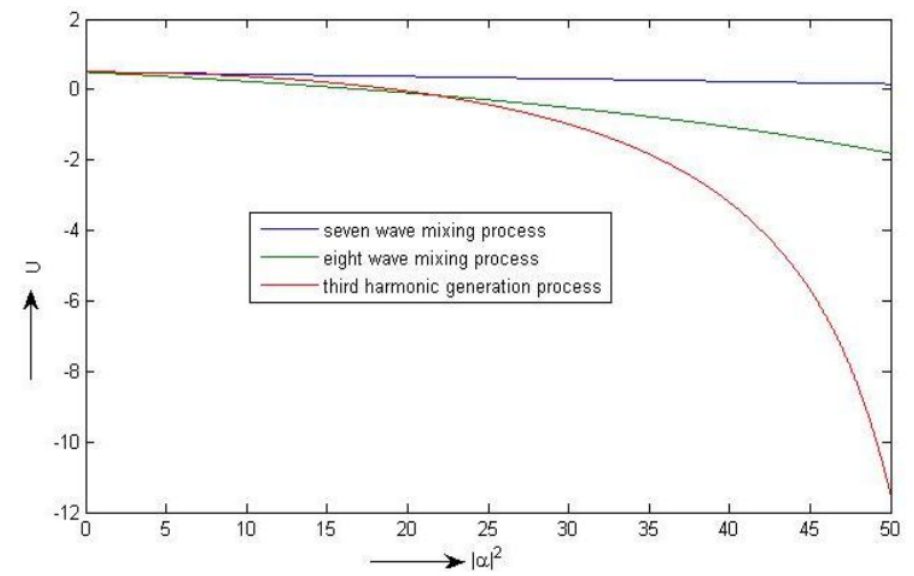

FIG. 1. Variation of phase fluctuation parameter $U$ with $|\alpha|^{2}$ for seven wave mixing, eight wave mixing and for third harmonic generation (taking $g^{2} t^{2} \approx 10^{-4}$ )

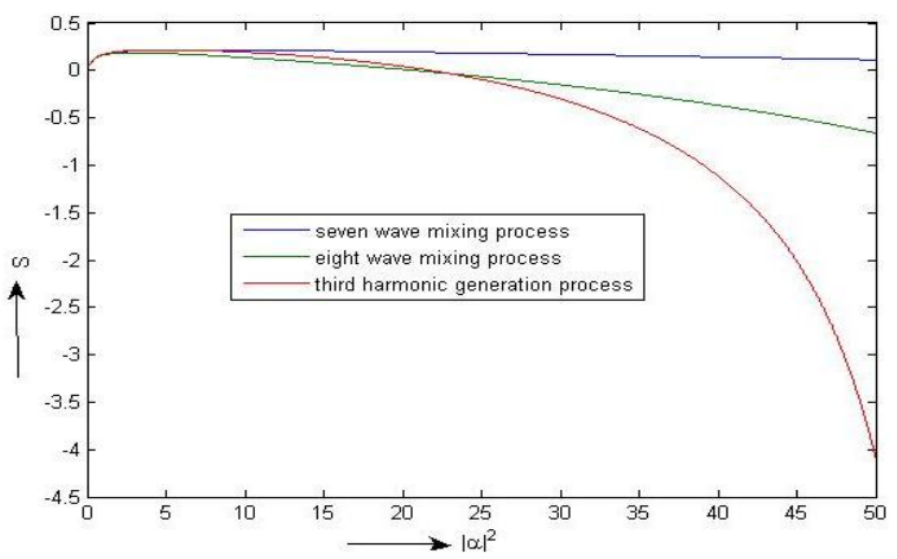

FIG. 2. Variation of phase fluctuation parameter $S$ with $|\alpha|^{2}$ for seven wave mixing, eight wave mixing and for third harmonic generation (taking $g^{2} t^{2} \approx 10^{-4}$ and $\theta=0$ )

Figures 4 and 5 show the variation of antibunching and minimum total noise with $|\alpha|^{2}$ and we observe that more is the antibunched state (sub-Poissonian), more will be the minimum total noise present in the system.

Thus we can conclude that the depth of non-classicality can be directly measured by using the quantum parameters i.e. phase fluctuation, antibunching and minimum total noise present in the system. 


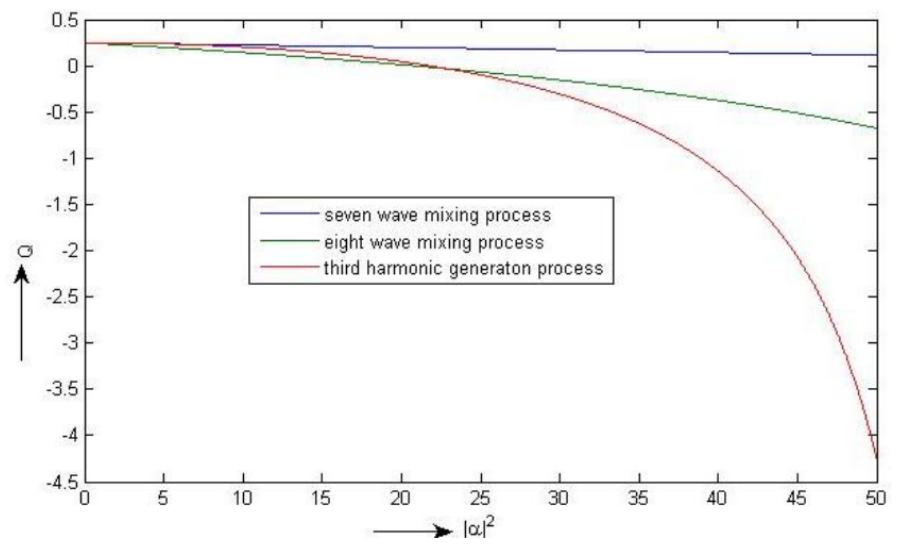

FIG. 3. Variation of phase fluctuation parameter $Q$ with $|\alpha|^{2}$ for seven wave mixing, eight wave mixing and for third harmonic generation (taking $g^{2} t^{2} \approx 10^{-4}$ and $\theta=0$ )

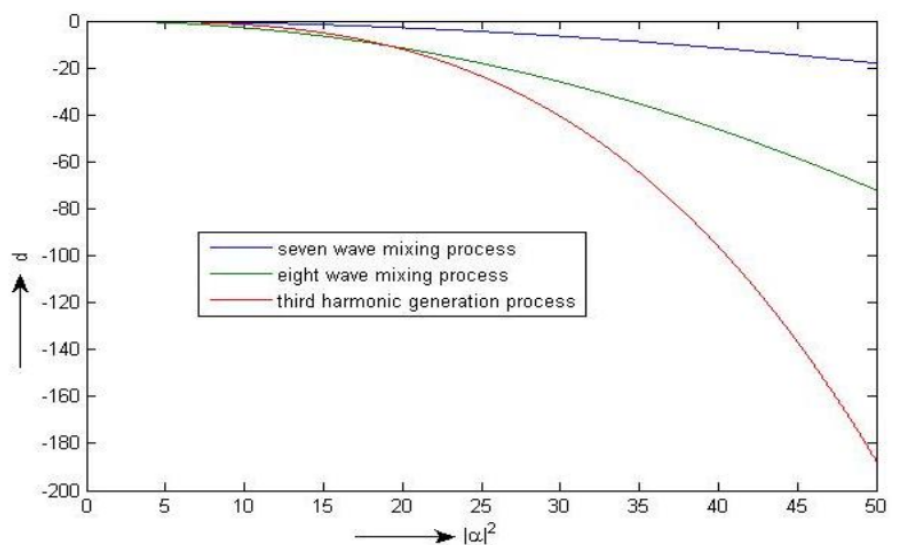

FIG. 4. Variation of $d$ with $|\alpha|^{2}$ for seven wave mixing, eight wave mixing and third harmonic generation (taking $g^{2} t^{2} \approx 10^{-4}$ )

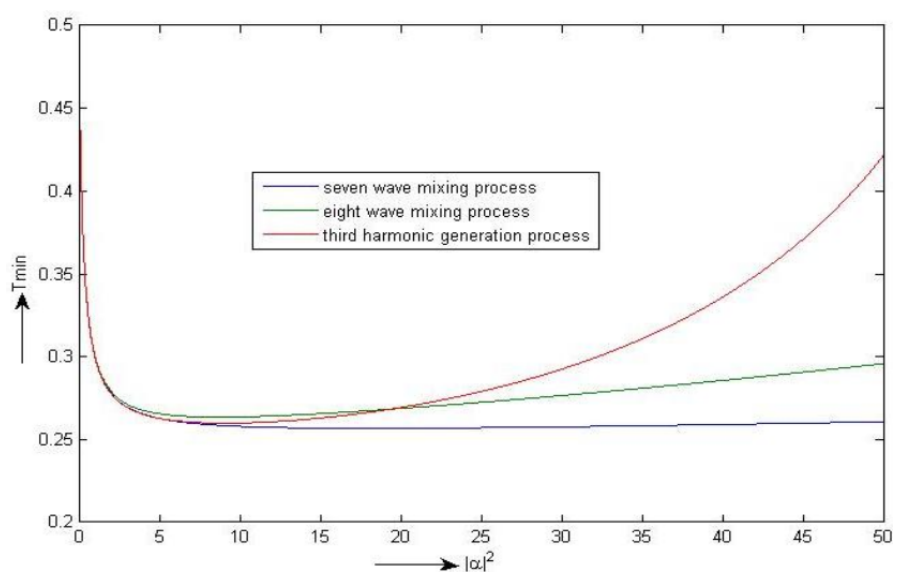

FIG. 5. Variation of $T_{\min }$ in antibunched (sub-Poissonian) state with $|\alpha|^{2}$ for seven wave mixing, eight wave mixing and for third harmonic generation (taking $g^{2} t^{2} \approx 10^{-5}$ ) 


\section{References}

[1] Meystre P., Sargent III M. Elements of quantum optics, Second ed., Springer, Berlin, 1999.

[2] Dodunov V.V. Theory of nonclassical states of light. Monko V.I. (Eds.), Taylor and Francis, New York, 2003.

[3] Orlowski A. Classical entropy of quantum states of light. Phyical Review A, 1993, 48 (1), P. 727-731.

[4] Lynch R. The quantum phase problem: a critical review. Physical Review, 1995, 256 (6), P. 367-436.

[5] Perinova V., Luks A., Perina J. Phase in optics, Chapter 4. World Scientific, Singapore, 1998.

[6] Ficek Z., Wahidd M.R. Quantum optics fundamental and applications, Chapter 4. IIUM Kuala Lumppur, 2004.

[7] Barnett S.M., Pegg D.T. Phase in quantum optics. Journal of Physics A: Mathematical and General, 1986,19 (18), P. 3849-3862.

[8] Pegg D.T., Barnett S.M. Phase properties of the quantized single model electromagnetic field. Physical Review A, 1989,39 (4), P. $1665-1675$.

[9] Susskind L., Glogower J. Quantum mechanical phase and time operator. Physics Physique Fizika, 1964, 1 (1), P. 49-61.

[10] Vaccaro J.A., Pegg D.T. On measuring extremely small phase fluctuations. Optics Commnication, 1964, 105 (5-6), P. 335-340.

[11] Lynch R. Phase fluctuations in the coherent light anharmonic oscillator systems via measured phase operators. Optics Communication, 1988, 67 (1), P. 67-70.

[12] Pegg D.D., Vaccaro J.A. Phase properties of squeezed states of light. Optics communication, 1989, 70 (6), P. $529-534$.

[13] Sanders C., Barnett S.M., Knight P.L. Phase variables and squeezed states. Optics Communication, 1986, 58 (4), P. $290-294$.

[14] Tsui Y.K. Josephson tunneling between superconductors in the angle-operator formalism. Physical Review B: Condensed Matter, 1993, 47 (18), P. 12296-12299.

[15] Lynch R. Phase fluctuations in a squeezed state using measured phase operators. Optical Society of America B, 1987,4 (10), P. $1723-1726$.

[16] Carruthers P., Nieto M.M. Phase and angle variables in quantum mechanics. Review Modern Physics, 1968,40 (2), P. $411-440$.

[17] Yao D. Phase properties of squeezed states of light. Phys. Lett. A, 1987, 122 (2), P. 77-83.

[18] Fan H.Y., Zaidi H.R. An exact calculation of the expectation values of phase operators in squeezed states. Optics Communication, 1988, 68 (2), P. 143-148.

[19] Pathak A., Mandal S. Phase fluctuations of coherent light coupled to a nonlinear medium of inversion symmetry. Physics Letter A, 2000, 272 (5-6), P. 346-352.

[20] Verma A., Pathak A. Reduction of quantum phase fluctuation in intermediate states. Physics Letter A, 2009,373 (16), P. 1421-1428.

[21] Kreibig U., Vollmer M. Optical properties of metal clusters. Springer Series in Materials Science, 1995, 25, P. $278-279$.

[22] Seongmin J.U., Wateker P.R., Jeong S. Nonlinear optical properties of zinc doped germane silicate glass optical fibre. Journal Nonlinear Optical Physics Matter, 2011, 19, P. 791-799.

[23] Xie R.H. Handbook of advanced electronic and photonic materials and devices. H.S. Nalwa (Ed.), Elsevier, 2000,9, P. $267-307$.

[24] Xie R.H., Rao Q., Jensen L. Encyclopedia of nanoscience and nanotechnology, H.S. Nalwa (Ed.), Elsevier, 2003.

[25] Stentz A.J., Boyd R.W. Hanbook of photonics, M.C. Gupta (Ed.), Chapter 5. CRC Press, 1997.

[26] Wang Q., Jianfeng Xu, Xie R.H. Nonlinear optics of nanoparticles and nanocomposites. Encyclopedia of nanoscience and nanotechnology, H.S. Nalwa (Ed.), 2004, 8, P. 101-111.

[27] Danilin S., Lebedev A.V. Quantum enhanced magnetometry by phase estimation algorithms with a single artificial atom. NPJ Quantum Information, 2018, 4, P. 1-7.

[28] Chakraverty B.K. Quantum phase fluctuation in high T) $c$ superconductors. Physica C, 2000, 341-348 (1), P. 75-78.

[29] Radkevich A., Semenov A.G., Zaikin A.D. Quantum phase fluctuations and density of states in superconducting nanowires. Physical Review $B, 2017,96(8), 085435$

[30] Faccioli M., Salasnich L. Gaussian quantum fluctuations in thesuperfluid Mott insulator phase transition. Physical Review A, 2019, 99 (2), 023614.

[31] Hillery M., Total noise and nonclassical states. Physical Review A, 1989, 39 (6), 2994. 